\title{
Attitudes to cataract surgery during the COVID-19 pandemic: a patient survey
}

\author{
Khayam Naderi $\circledast^{1,2} \cdot$ Laura Maubon $^{1} \cdot$ Ashmal Jameel $^{1} \cdot$ Darshak S. Patel $^{1} \cdot$ Jack Gormley $^{1} \cdot$ Vishal Shah $^{1} \cdot$ Lily Lai $^{3}$. \\ Sancy Low ${ }^{1} \cdot$ Seema Verma ${ }^{1} \cdot$ Scott Robbie ${ }^{1} \cdot$ Mani Bhogal ${ }^{1} \cdot$ David O'Brart $\mathbb{B}^{1,2}$
}

Received: 20 June 2020 / Revised: 14 July 2020 / Accepted: 16 July 2020 / Published online: 24 July 2020

(c) The Royal College of Ophthalmologists 2020

We applaud Shih et al. [1] initiatives in ensuring continuity of clinical care for ophthalmology patients at the University of Hong Kong during the current COVID-19 pandemic. Taking into account the available guidance on infection control measures, their efforts to ensure that both patients and clinical staff remain safe in clinical settings is to be commended. However, in addition to the substantial impact of COVID-19 on healthcare in general [2], one of the consequences of lockdown measures in the United Kingdom and elsewhere has been a significant reduction in elective surgery, including cataract surgery (CS). CS is the commonest elective surgery in the National Health Service (NHS) with good postoperative and quality of life (QOL) improvements [3, 4]. There is no published evidence regarding attitudes and concerns of patients awaiting elective surgery during the current pandemic. The median age of CS patients in the UK is 76.3 years [3], placing them in a vulnerable COVID-19 age group. The selection of patients for CS, with consideration of their concerns and expectations, is important for direct patient care and wider healthcare planning. To address this, we undertook a questionnaire survey to explore patient attitudes and concerns towards undergoing CS during this pandemic.

The survey was approved by our institution's audit and quality improvement project team. Data collection adhered to the tenets of the Declaration of Helsinki and UK Data Protection Act. The patient sample included all patients on the CS waiting list $(n=376)$ at our institution. Patients were

David O'Brart

davidobrart@gstt.nhs.uk

1 Department of Ophthalmology, St. Thomas' Hospital, Lambeth Palace Road, London SE1 7EH, United Kingdom

2 King's College, London WC2R 2LS, United Kingdom

3 Department of Ophthalmology, King's College Hospital, Normanby Building, Denmark Hill, London SE5 9RS, United Kingdom contacted by telephone from 20th May to 5th June 2020. Informed verbal consent was obtained, and an anonymized seven-question survey completed for each patient. A 5-point Likert scale was used for each question, and each question was analysed independently. Data were analysed using SPSS Version 26.0 (IBM Corp, Armonk, NY, USA). Mann-Whitney and Spearman Rank Correlation tests were used to compare non-parametric data.

Two attempts were made to contact each patient. The survey was completed by 207 (55\%). Patient demographics and responses are displayed in Table $1.64 .8 \%$ of patients strongly agreed/agreed that their eyesight was reducing their QOL. 70\% were prepared to attend hospital for CS within one month. $27.6 \%$ preferred to wait until there were no more cases of COVID-19 or a vaccine developed, even if this was over 6 months. 30.9\% responded that they would not attend as the person accompanying them on the day of surgery was worried about COVID-19. $47.3 \%$ and $37.2 \%$ were concerned about contagion in the hospital or on their journey to/from hospital, respectively. $26.6 \%$ were more positive towards immediate sequential bilateral cataract surgery (ISBCS), which might help surgical throughput [5]. There was a positive correlation between worsening QOL and willingness to attend $(r=0.236$, $p=0.001$ ). Patients were less likely to attend if they were worried about contagion in hospital $(r=-0.487, p=0.000)$ or on their journey $(r=-0.413, p=0.000)$, or if the accompanying person was concerned $(r=-0.458, p=0.000)$. There were no differences in responses between genders, first or second eye CS patients, and under-70 and over-70 age groups. There were no differences between responders/non-responders in terms of age, gender or first or second eye surgery.

At a time when UK lockdown measures are only starting to be eased, with substantial new daily case numbers and the highest reported numbers of deaths in Europe [2], 70\% of our patients, many of whom are age vulnerable, are willing to attend for elective CS. Indeed $64.8 \%$, whose surgery has been delayed, feel that their QOL has deteriorated due to worsening eyesight, reflecting the indirect effects on morbidity of 
Table 1 Patient demographics and responses to questionnaire survey.

\begin{tabular}{ll}
\hline Patient demographics & $n(\%)$ \\
\hline Survey responses & 207 \\
Males:Females & $87: 120$ \\
Age range (years) & $41-94$ \\
Mean age (years) & 69.6 \\
Waiting for 1st Eye: 2nd Eye & $105: 102$
\end{tabular}

Waiting for 1st Eye: 2nd Eye

105:102

1. My eyesight is significantly interfering with my quality of life.

Strongly agree

Agree

Neutral

Disagree

Strongly disagree

2. I am happy to come in within the next month for my cataract surgery

Strongly agree

Agree

Neutral

Disagree

Strongly disagree

3. I wish to come in for my cataract surgery until there are no more cases of Coronavirus in the UK and/or there is a vaccine for Coronavirus, even if this takes 6 months or next year.

Strongly agree

$26(12.6 \%)$

$31(15.0 \%)$

$16(7.7 \%)$

$55(26.6 \%)$

$79(38.2 \%)$

Strongly disagree

4. I cannot come in for cataract surgery as my family member/carer/friend who has to accompany me on the day is worried about Coronavirus.

Strongly agree

Agree

Neutral

Disagree

Strongly disagree

5. I am worried about contracting Coronavirus at the hospital when having my cataract surgery.

Strongly agree

$45(21.7 \%)$

Agree

Neutral

Disagree

Strongly disagree

6. On the day of cataract surgery, I am worried about catching Coronavirus on my journey to and from hospital.

Strongly agree

$34(16.4 \%)$

$43(20.8 \%)$

$13(6.3 \%)$

$21(10.1 \%)$

$33(15.9 \%)$

$55(26.6 \%)$
Table 1 (continued)

\begin{tabular}{ll}
\hline Patient demographics & $n(\%)$ \\
\hline Disagree & $46(22.2 \%)$ \\
Strongly disagree & $71(34.3 \%)$ \\
7. My attitude to the idea of having cataract surgery for & \\
both of my eyes in the same sitting has changed during & \\
the pandemic. & $25(12.1 \%)$ \\
Strongly agree & $30(14.5 \%)$ \\
Agree & $77(37.2 \%)$ \\
Neutral & $36(17.4 \%)$ \\
Disagree & $39(18.8 \%)$ \\
Strongly disagree &
\end{tabular}

measures required to prevent COVID-19. This coupled with the likely substantial numbers of individuals in the community with visually-significant cataract, undiagnosed because of the lockdown measures and now requiring CS, suggests that the recommencement of an adjusted, safe, elective CS service with sufficient case numbers to meet demand and prevent excessive future CS waiting times is imperative. It is, however, of note that almost half of our patients and $30 \%$ of their relatives/carers/friends are concerned about the risks of contracting COVID-19 at the hospital and/or during their journey and they will require reassurance that all possible precautions and protocols are being undertaken to prevent contagion.

Data sharing and declaration DOB holds non-commercial research grants with Rayner Ltd. and Avedro Inc. He has undertaken consultancy work for Avedro Inc, Sparca Inc. and Alcon Inc. in the past 12 months. He holds stock in Sparca Inc. SR has undertaken consultancy work for Invua MedTech Ltd.

Publisher's note Springer Nature remains neutral with regard to jurisdictional claims in published maps and institutional affiliations.

\section{References}

1. Shih CK, Chan JCH, Lai JSM. Maintenance of ophthalmic specialist out-patient service during the COVID-19 outbreak: The University of Hong Kong experience. Eye (Lond). 2020;34: 1241-2. https://doi.org/10.1038/s41433-020-0887-z.

2. WHO Coronavirus Disease (COVID-19) Dashboard. https:// covid19.who.int/?gclid=EAIaIQobChMIxrjhn_rO6QIVNoBQBh3 QdgBtEAAYASAAEgInYvD_BwE.

3. National Ophthalmology Database Audit. 2019. https://www.noda udit.org.uk/u/docs/20/urxqilwxmv/NOD\%20Audit\%20Annual\% 20Report\%202019.pdf.

4. Lamoureux EL, Fenwick E, Pesudovs K, Tan D. The impact of cataract surgery on quality of life. Curr Opin Ophthalmol. 2011;22:19-27.

5. O’Brart D, Roberts H, Naderi K, Gormley J. Economic Modelling of Immediately Sequential Bilateral Cataract Surgery (ISBCS) in the National Health Service based on possible improvements surgical efficiency. BMJ Open Ophth. 2020;5:e000426 https://doi.org/ 10.1136/bmjophth-2019-000426. 